\title{
Magnetic Skyrmions Could Act as Qubits
}

\section{Predictions suggest that magnetic quasiparticles known as skyrmions could provide the basis for a novel quantum-computing platform.}

\section{By Marric Stephens}

$\mathrm{n}$ the race to build a useful quantum computer, superconducting qubits currently lead the field of possible building blocks for the devices. Now researchers propose a new challenger for this role: a topologically stabilized magnetic feature called a skyrmion. The idea of using skyrmions as qubits has been suggested by Christina Psaroudaki at the California Institute of Technology and Christos Panagopoulos at Nanyang Technological University in Singapore, who say that this system should be scalable and easy to manipulate [1].

Skyrmions are vortex-like quasiparticles that form when the local orientation of a magnetic material's atomic spins deviates from that of the background. The quasiparticles are described by two key parameters: their topological charge (an integer that indicates how many times magnetic moments within a skyrmion wrap a sphere) and their helicity (a variable that indicates the angle of the spin axes from the crystallographic axis).

The scheme proposed by Psaroudaki and Panagopoulos is

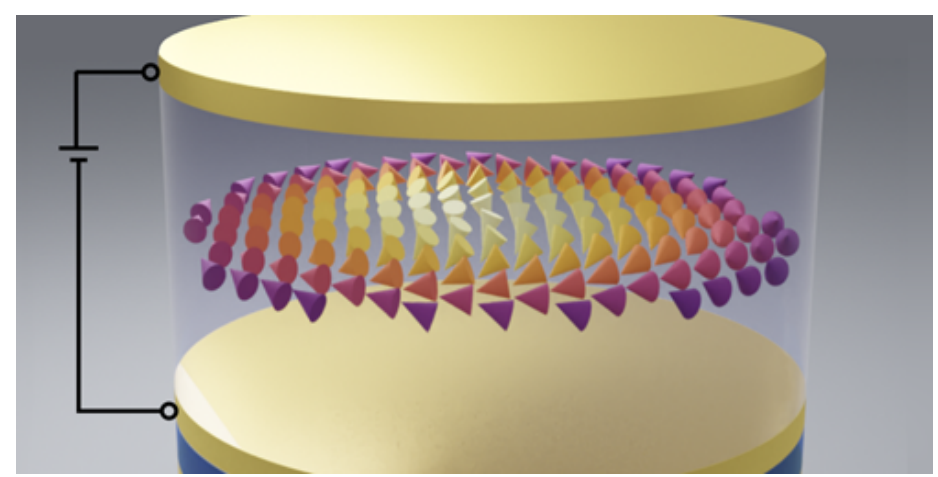

Credit: C. Psaroudaki and C. Panagopoulos [1] based on skyrmions stabilized in magnetic nanodisks bounded by electrical contacts. Static electric and magnetic fields control the quantized energy spectra of the skyrmions, allowing the user to change the helicity between two energetically favored levels, which act as a qubit's 0 and 1 states. These electric and magnetic fields can also be tuned to control the lifetimes of the qubits. The qubits interact with their neighbors, which reside in adjacent nanodisks, via nonmagnetic spacers. Readout comes via sensitive magnetometers.

The duo has identified several candidate materials that have the necessary magnetic configuration and strong geometrical frustration to host skyrmion qubits. They expect more candidate materials to emerge as the field of skyrmionics advances.

Marric Stephens is a Corresponding Editor for Physics based in Bristol, UK.

\section{REFERENCES}

1. C. Psaroudaki and C. Panagopoulos, "Skyrmion qubits: A new class of quantum logic elements based on nanoscale magnetization," Phys. Rev. Lett. 127, 067201 (2021). 